\title{
The nonelastic reaction code BRIEFF and its intranuclear cascade BRIC
}

\author{
H. Duarte \\ CEA/DIF, BP. 12, 91680 Bruyères-le-Châtel, France
}

\begin{abstract}
The intranuclear cascade (INC) code of Bruyères-le-Châtel named BRIC is the first part of the nonelastic reaction code BRIEFF. Recent changes in our INC are presented. They were done for nucleon induced reaction to improve results below $100 \mathrm{MeV}$, and to calculate cross sections of compound nucleus formation. These cross sections are used in the evaporation component of our reaction code. BRIEFF is included in the Bruyères-le-Chatel version of HETC to perform thick targets calculations. BRIC has recently been incorporated into MCNPX 2.4.0 to verify thick target results of neutron yields and to do some comparisons with other nuclear models or libraries. Good agreement with data is obtained on average.
\end{abstract}

\section{Introduction}

The intranuclear cascade BRIC is described in ref. [1]. In the current paper we present some improvements on calculation at low intermediate energy, between threshold and $100 \mathrm{MeV}$. Such calculations are useful not only to get more reliability in the energy-angle distribution of low energy outgoing nucleons that are present at any moment of the INC stage, but also to calculate cross sections necessary in the evaporation model. Those cross sections of compound nuclei formation are now used in the code BRIEFF. In order to perform thick target calculations, BRIEFF is included into HETC-BRC, the Bruyères-le-Châtel version of HETC. We present some of its thick target results and also when the current version of BRIC is incorporated into MCNPX 2.4.0 [2].

\section{Current version of BRIC}

In BRIC, the nucleon spatial distribution of the target nucleus follows a Woods-Saxon function whose parameters were fitted on theoretical calculations. The spherical nucleus potential $V(r)$ is the sum of a nuclear potential $V_{N}$ derived from the Fermi gas approximation and of a Coulomb potential $V_{C}$. During the INC calculation, hadrons (nucleons, pions and $\Delta$ here) are points in phase space separated into two species, the quasifree hadrons and the bound nucleons. A bound nucleon at position $\boldsymbol{r}-\boldsymbol{r}_{G}$ from the center of mass of the nucleus $\boldsymbol{r}_{G}$ has a kinetic energy lower than $\operatorname{Max}\left(-V\left(\left|\boldsymbol{r}-\boldsymbol{r}_{G}\right|\right), 0\right)$. Other hadrons are quasifree particles. The position $\left\{\boldsymbol{r}_{i}, \boldsymbol{p}_{i}\right\}$ of a quasifree hadron follows the equations of motion

$$
\begin{aligned}
\frac{d \boldsymbol{p}_{i}}{d t} & =-\boldsymbol{\nabla}_{r_{i}} V\left(\left|\boldsymbol{r}_{i}-\boldsymbol{r}_{G}\right|\right), \\
\frac{d \boldsymbol{r}_{i}}{d t} & =\frac{\boldsymbol{p}_{\boldsymbol{i}}}{E_{i}} .
\end{aligned}
$$

On the other hand a bound nucleon stays static in phase space as long as it has not be strucked by a quasifree hadron or when its kinetic energy becomes less than $\operatorname{Max}\left(-V\left(\mid \boldsymbol{r}_{i}-\right.\right.$

\footnotetext{
${ }^{a}$ e-mail: helder.duarte@cea.fr
}

$\left.\boldsymbol{r}_{G} \mid\right), 0$ ) inside nucleus. Spatial positions, energy-momentum of the hadrons and of the nucleus and other quantities like excitation energy of the nucleus or $V_{C}$ change with the INC time. The collision term is detailled in refs. [1] and [3], we only underline here that the in-medium cross section of $\mathrm{Li}$ and Machleidt [4] allowed us to improve calculations at low intermediate energy [1].

In BRIC 1.4, the INC simulation of nonelastic events starts by the initialisaton of the incident hadron at a position $\boldsymbol{r}_{\text {ini }}=R_{\text {limit }}$ and a momentum $\boldsymbol{p}_{\text {ini }}=p \cdot \boldsymbol{u}_{z}$ ( $p$ depends on the incident energy $e_{i n i}$ and on the potential at $\boldsymbol{r}_{i n i}, \boldsymbol{u}_{z}$ being the incident direction at infinity; $R_{\text {limit }}$ is the maximal radius defined in [1]). This initialisation $\left\{\boldsymbol{r}_{i n i}, \boldsymbol{p}_{\text {ini }}\right\}$ is wrong at low intermediate energy and it worsens when $e_{i n i}$ decreases and when the charge $Z$ of the target nucleus increases. To correct this discrepancy, equations of motion (1) and (2) are computed from $5 \times R_{\text {limit }}$ to $R_{\text {limit }}$ before the INC simulation. It improves noticeably the calculation of the reaction cross section as it will be shown latter.

In BRIC 1.4 the production of particle was also too low for nucleon induced reaction on heavy target nuclei, below $100 \mathrm{MeV}$. Blue dashed histograms in figure 1 shows the underproduction of higher outgoing energy neutrons in proton induced reactions on ${ }^{208} \mathrm{~Pb}$. To correct this discrepancy the geometrical parameters, radius $R_{V}$ and diffuseness $a_{V}$, of the nuclear potential $V_{N}(r)$, were differentiated from those of the matter density $\rho(r)$. Constant variation was assumed: $R_{V}=R(A)+d R_{V}$ and $a_{V}=a(A)+d a_{V}$, where $R(A)$ and $a(A)$ are the mass dependent parameters of $\rho(r)$.

To keep a continuity with our previous work, the potential shape of $V_{N}(r)$ stays proportionnal to $\rho(r)^{2 / 3}$ and the depth $V_{0}$ is still fixed to $-48 \mathrm{MeV}$ as before. Some values of $d R_{V}$ and $d a_{V}$ were tested by comparing BRIC results with differential cross section of particle production for reactions on some light, medium and heavy nuclei. The empirical parameters $d R_{V}=0.8 \mathrm{fm}$ and $d a_{V}=0.06$ appear to be a good compromise for all target mass but they are more preliminary than final parameters. We notice nevertheless that their positive values are compatible with a nuclear potential that would come from the folding of the matter density with a finite range function. We postpone a more complete study of the potential to a forthcoming publication. The red solid histograms in 


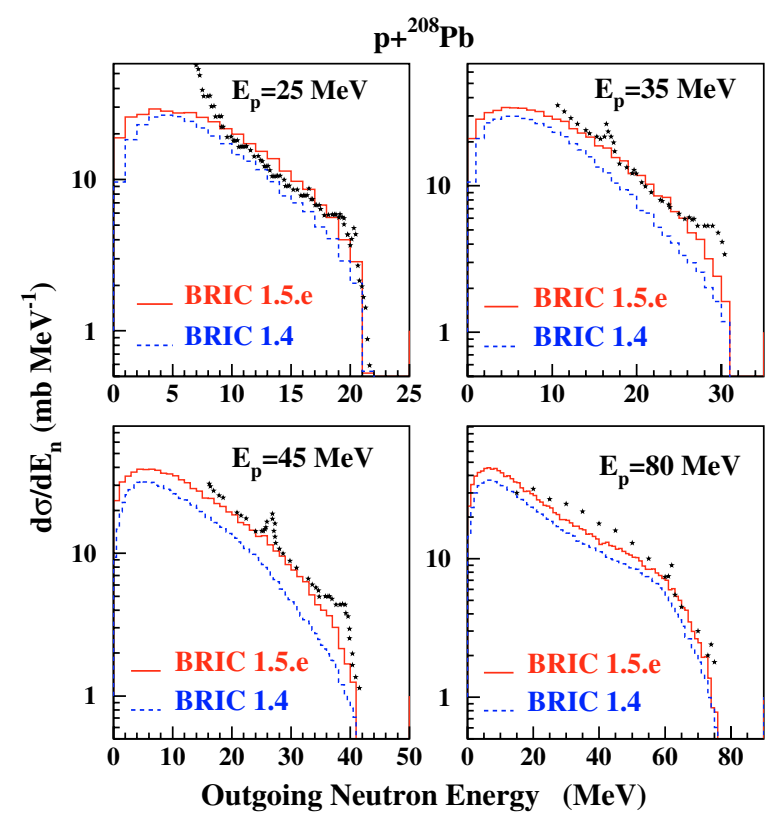

Fig. 1. Differential cross section of neutron production in proton induced reaction on ${ }^{208} \mathrm{~Pb}$. Black symbols are data from [5].

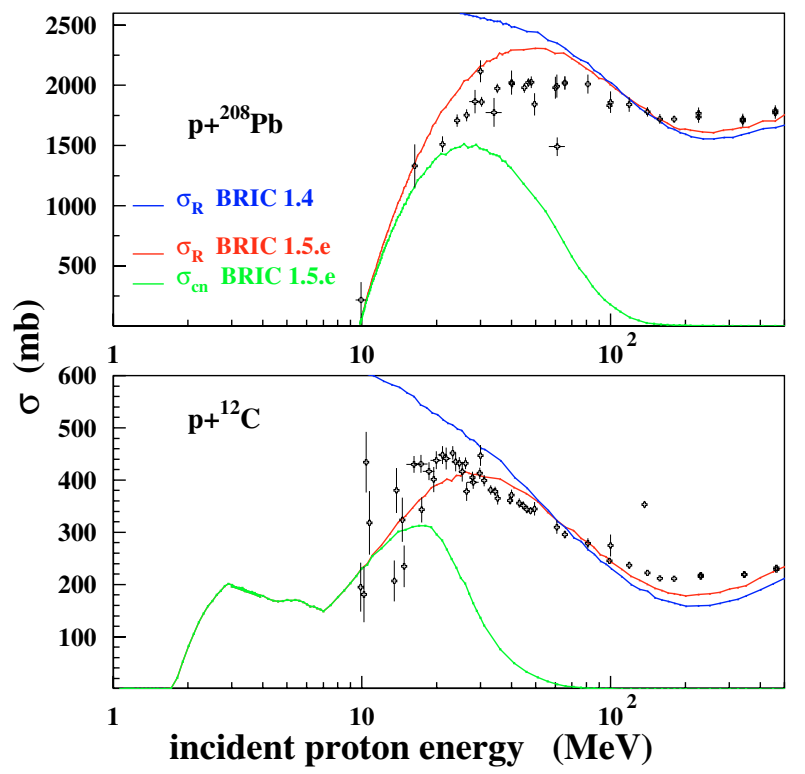

Fig. 2. Reaction cross section for $\mathrm{p}+{ }^{208} \mathrm{~Pb}$ and $\mathrm{p}+{ }^{12} \mathrm{C}$. Red and blue curves are results of BRIC 1.5.e and 1.4, respectively. Green solid curve is the cross section of compound nucleus formation obtained with BRIC 1.5.e. Experimental data comes from the EXFOR database [6].

figure 1 are the results of the current version of BRIC in better agreement with data than the previous one.

With the improvement of the initialisation, Coulomb+ nuclear (nuclear) deflection of low incident energy protons (neutrons) is now taken into account. Figure 2 shows the reaction cross sections of $\mathrm{p}+{ }^{208} \mathrm{~Pb}$ and $\mathrm{p}+{ }^{12} \mathrm{C}$ compared to experimental data. The results of BRIC 1.5.e are in better agreement with data at low intermediate energy.

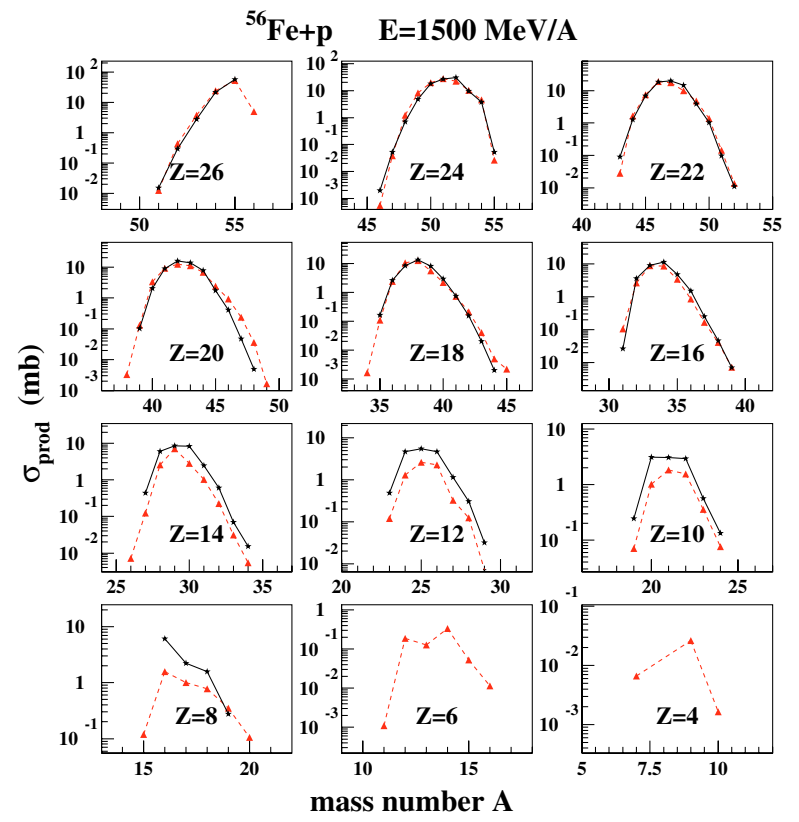

Fig. 3. Production cross section of residual nuclei in ${ }^{56} \mathrm{Fe}+$ proton reaction at $1500 \mathrm{MeV} / \mathrm{A}$. Only residual nuclei of even $\mathrm{Z}$ are shown here. Black symbols are data from [10].

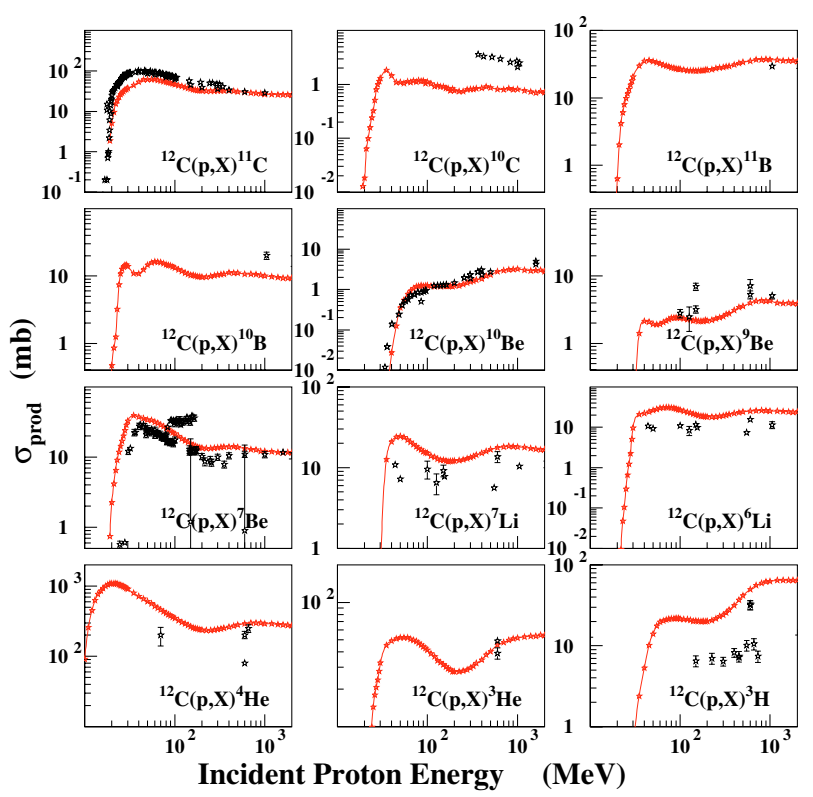

Fig. 4. Excitation function of production cross section in $\mathrm{p}+{ }^{12} \mathrm{C}$ reaction between 10 and $2000 \mathrm{MeV}$. Black symbols are experimental data extracted from EXFOR database [6].

The improvement between threshold and $100 \mathrm{MeV}$ presents another advantage: a cross section useful to our evaporation model can now be extracted from BRIC calculations.

\section{Description of BRIEFF}

In the statistical theory of Weisskopf-Ewing [7] based on time reversibility, the probability of emission of particle $b$ and the distribution of its kinetic energy depend on the cross section 


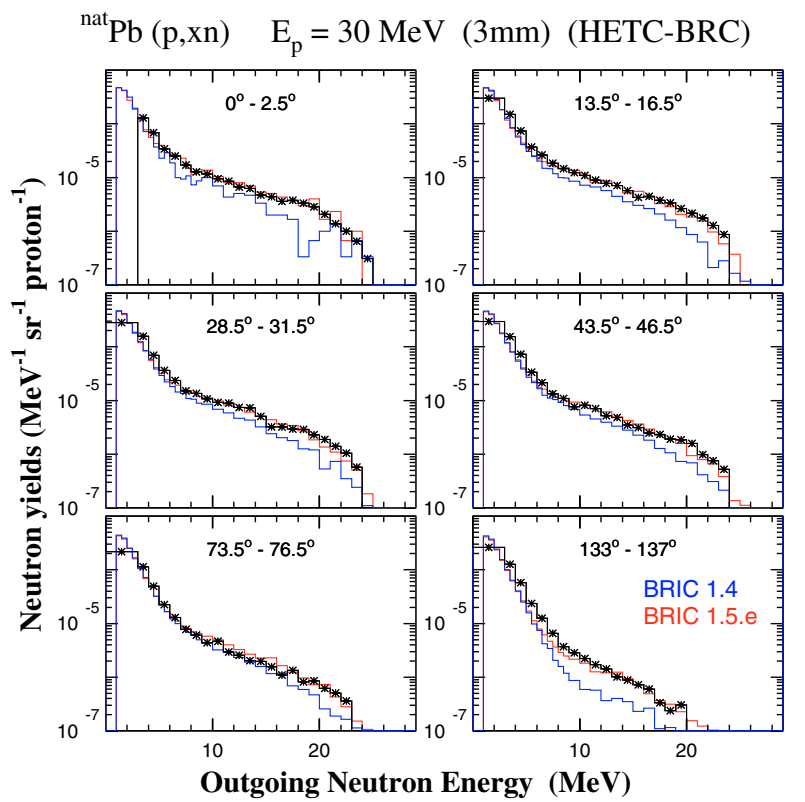

Fig. 5. Neutron yields at 6 angles for $30 \mathrm{MeV}$ proton on ${ }^{\text {nat }} \mathrm{Pb}(3 \mathrm{~mm}$ thickness). HETC-BRC calculations were done with BRIC 1.4 (blue histograms) and BRIC 1.5.e (red histograms). Angular intervals of calculation are indicated. Black symbols are data from ref. [12].

of the compound nucleus formation $\sigma_{c n}^{(b)}=\sigma\left({ }^{a_{b}} b_{z b}+{ }^{A} X_{Z} \rightarrow\right.$ $\left.{ }^{A+a_{b}} Y_{Z+z b}^{*}\right)$. At low energy it can be identified as the reaction cross section. However as the incident energy increases, an increasing part of the reaction cross section goes into the channel of "preequilibrated" particles emission that must not enter into the calculation of $\sigma_{c n}^{(b)}$. In BRIC this cross section is the fraction of nonelastic events for which no emission occurs before the time cut of INC is reached. For proton induced reactions of figure 2 they are the green curves that rapidly decrease after 20-30 MeV. In order to use the cross sections in the evaporation of BRIEFF, calculations were performed for 2000 target nuclei and for incident neutron and proton.

For $\mathrm{d}, \mathrm{t},{ }^{3} \mathrm{He}$ and $\alpha$ emission, BRIC still needs some developments to fully complete the fast stage of reaction induced by light charged particles. For the moment, the cross sections of compound nucleus formation are the previous reaction cross sections from Glauber calculations [1] multiplied by $\sigma_{c n}^{(p)} / \sigma_{R}^{(p)}$ of proton BRIC results shifted to the threshold energy of light charged particle induced reaction.

The other parts of the de-excitation stage are the fission of heavy nuclei and the fragmentation of excited light nuclei. Fission of heavy nuclei can occur in competition with evaporation of neutron and light charged particles. The fission model of Atchison [8] is used with a phenomenological parameter applied to the ratio of decay width $\Gamma_{f} / \Gamma_{n}$ since our evaporation model is different from the one used by Atchison. Evaporation of compound nucleus is replaced by the Fermi Breakup model when the mass of excited compound nucleus is less than 30 . Probability of 2- and 3-body decays are calculated with, in final state of decay, at least one light charged nucleus of mass $A \leq 10$ in one discrete state (up to 30 levels).

In figure 3 the production of residual nuclei from ${ }^{56} \mathrm{Fe}+\mathrm{p}$ reaction at $1500 \mathrm{MeV} / \mathrm{A}$ are compared to experimental data of

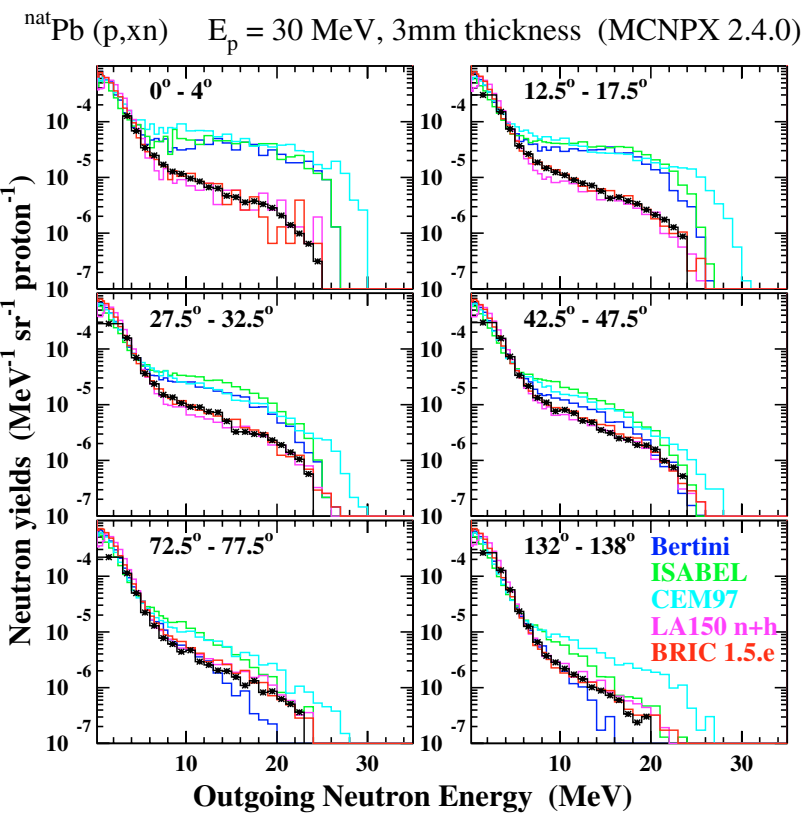

Fig. 6. Same as figure 5 for MCNPX 2.4.0 calculations (angular intervals are indicated). Default options of INCs are used.

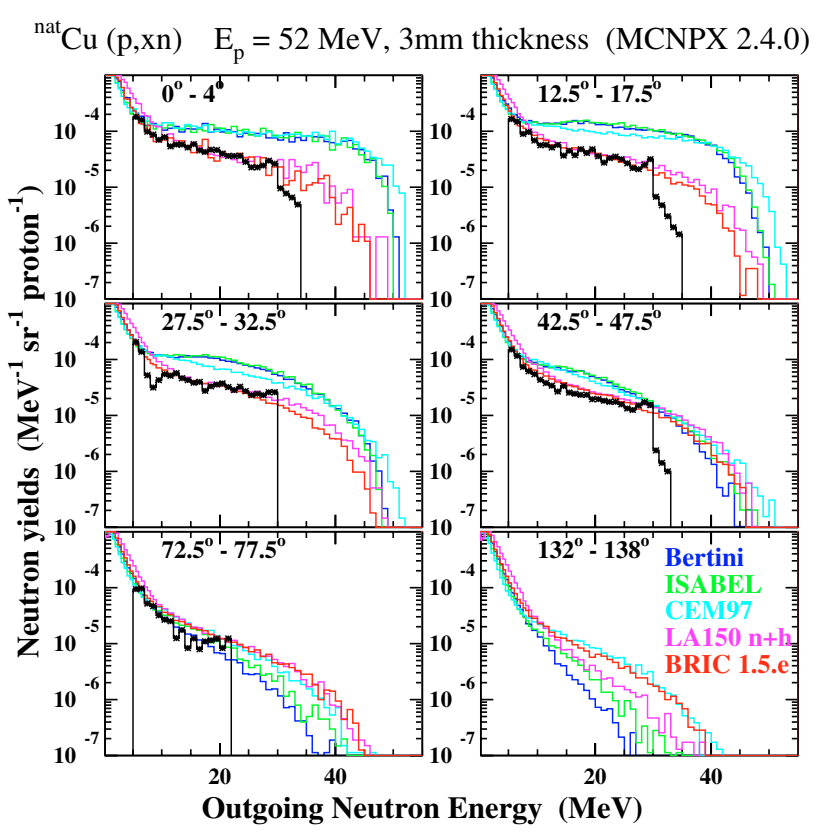

Fig. 7. Same as figure 6 for $52 \mathrm{MeV}$ proton on $3 \mathrm{~mm}$ thick copper target. Black symbols are data from [12]. No data are available at $135^{\circ}$.

ref. [10]. In figure 4 excitation functions of nuclei production for $\mathrm{p}+{ }^{12} \mathrm{C}$ reaction are compared to available data from 10 to $2000 \mathrm{MeV}$.

\section{Thick target calculation}

BRIEFF is included into HETC-BRC, a largely modified version of the original HETC [11], to generate nonelastic reactions in thick target simulation. They can be performed 


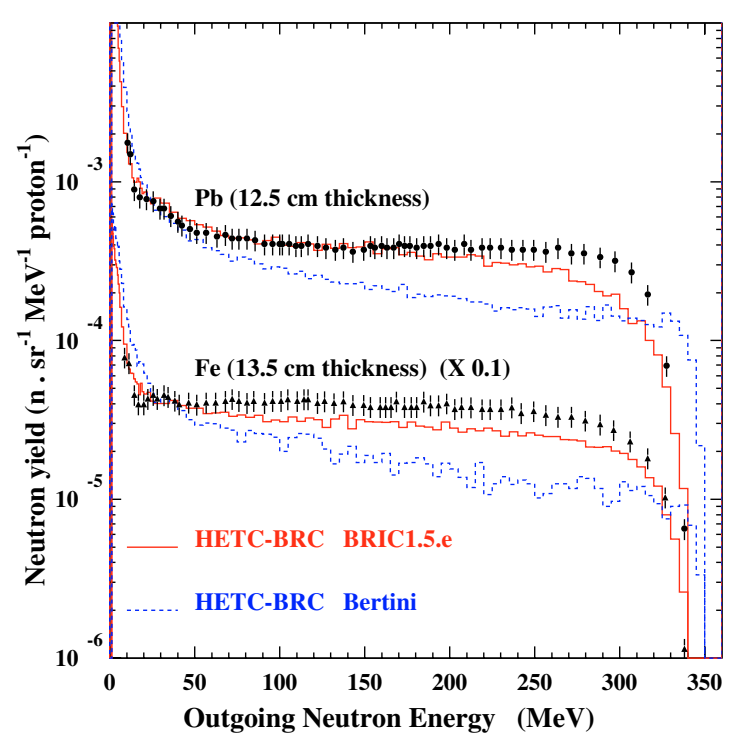

Fig. 8. Neutron yields at $0^{\circ}$ for $350 \mathrm{MeV}$ protons on targets of $\mathrm{Pb}$ $(12.5 \mathrm{~cm}$ thickness) and of $\mathrm{Fe}(13.5 \mathrm{~cm}$ thickness $)$ with HETC-BRC (angular interval is $0^{\circ}-2.5^{\circ}$ ). Black symbols are data from [14].

down to threshold energy. Nevertheless a cut at $1 \mathrm{MeV}$ is applied for neutrons to save time computation.

Figure 5 presents neutron yields from the irradiation of $3 \mathrm{~mm}$ thick lead target by $30 \mathrm{MeV}$ protons at 6 angles. BRIC 1.5.e results are in good agreement with data. Beam protons are stopped inside target. With the proton energy loss it means that nonelastic reactions of beam protons contributing to neutron production are done between $30 \mathrm{MeV}$ and $\sim 10 \mathrm{MeV}$. To verify the quality of such thick target calculations, BRIC has been incorporated into MCNPX 2.4.0. Figure 6 presents the same calculation performed with this transport code. Results with other INC models of MCNPX 2.4.0 and with the LA150 $\mathrm{n}+\mathrm{h}$ libraries [13] are also shown. We verified with other targets and other energy (see fig. 7) that BRIC 1.5.e results are in rather good agreement with experimental data and also with the results obtained with the LA150 $n+h$ libraries. The other INC models in MCNPX 2.4.0 overestimate the neutron production at forward angles in this energy range.

To confirm the quality of BRIC calculation, reactions at higher energies were performed. Figure 8 presents neutron yields at $0^{\circ}$ (beam direction) from the irradiation of thick iron and lead targets by $350 \mathrm{MeV}$ protons. HETC-BRC results with the INC of Bertini is also shown. It gives an idea of the trend of other calculations presented in ref. [14]. The forward emission of neutrons calculated by BRIC is again in better agreement with data than for other nuclear models.

\section{Conclusion}

The current version of BRIC calculates energy-angle and energy distributions of particle production in good agreement with data above $10-20 \mathrm{MeV}$. Near threshold most of the nonelastic reaction is the compound nucleus formation. It allows us to build a database for the evaporation component of BRIEFF. This database needs to be completed for heavier target nuclei however cross sections of residual nuclei production agree well with data on average for light and medium nuclei.

In the neutron production studies presented here, high outgoing energy neutrons come from the fast stage of the reaction, the INC, or from the libraries LA150 (but only below $150 \mathrm{MeV}$ ). HETC-BRC calculations with BRIC 1.5.e are rather good in thick target simulation. Since results of proton induced reaction depends also on the energy loss treatment in the transport code, we verified that contribution of BRIC in HETC-BRC calculations is right by incorporating our INC code into MCNPX 2.4.0. We noted that the results with our INC is in better agreement with data than the results of other INC models of MCNPX 2.4.0 at low intermediate energy, and in good agreement with the LA150 $\mathrm{n}+\mathrm{h}$ libraries. At higher energies our results are also of good quality. BRIC is then a solid basis for our nonelastic reaction code BRIEFF.

I would like to thank O. Bersillon for his support and for all the work done on HETC-BRC. I am also grateful to MCNPX team for the authorization to include our INC code into MCNPX. I also thank the staff of the Centre de Calcul Recherche et Technologie where this work has been partly performed.

\section{References}

1. H. Duarte, Phys. Rev C 75, 024611 (2007).

2. L.S. Waters (ed.), MCNPX User's Manual Version 2.4.0, Los Alamos National Laboratory report, LA-CP-02-408 (Sept. 2002).

3. H. Duarte, Proceedings of the Third International Conference on Accelerator-Driven Transmutation Technologies and Applications, Praha, Czech Republic, June 7-11, 1999.

4. G.Q. Li, R. Machleidt, Phys. Rev. C 48, 1702 (1993); Phys. Rev. C 49, 566 (1994).

5. M. Blann et al., Nucl. Phys. A 257, 15 (1976); M. Trabandt et al., Phys. Rev. C 39, 452 (1989).

6. http://www-nds.iaea.org/exfor/exfor00.htm

7. V.F. Weisskopf, D.H. Ewing, Phys. Rev. 57, 472 (1940).

8. F. Atchison, in Proceedings of a Specialists' Meeting, OECD/NEA, Issy-les-Moulineaux, France, 1994, p. 199.

9. E. Gradsztajn et al., Phys. Rev. Lett. 14, 436 (1965).

10. C. Villagrasa-Canton, Ph.D. thesis, Université de Paris XI, France, December 2003.

11. T.W. Armstrong, K.C. Chandler, Nucl. Sci. Eng. 49, 110 (1972).

12. T. Nakamura, M. Yoshida, K. Shin, Nucl. Instrum. Meth. 151, 493 (1978).

13. M.B. Chadwick et al., Nucl. Sci. Eng. 131, 293 (1999).

14. Y. Iwamoto et al., Nucl. Instrum. Meth. A 562, 789 (2006). 